\title{
INDICATIVO POR SUBJUNTIVO EN CLÁUSULA REGIDA POR EXPRESIÓN DE REACGIÓN PERSONAL
}

\section{Propósito y CORPUS DEL ESTUdio}

Es bien sabido que la expresión de reacción personal, tanto de tipo emotivo (por ejemplo, "alegrarse", etc.) como de tipo valorativo ("gustar", etc.), exige subjuntivo en cláusula que rige1. De ahí que se dice, por ejemplo, "Me alegro de que / Me gusta que estés aquî", y no "Me alegro de que / Me gusta que *estás aquí". Gili y Gaya (1964, § 110), por ejemplo, afirma lo anterior explicando que se emplea el subjuntivo en una cláusula regida por un verbo de emoción, afírmese o no la realidad del hecho ("Tengo miedo de que lleguen tarde", "Me duele que sea tan malo"). Vogel (1979, pp. 22-25) declara en forma perentoria que es inadmisible el empleo del indicativo en tal caso:

It is well known that the subjunctive occurs in a sentence embedded under a verb, adjective or noun that expresses a feeling or emotion. The inadmissibility of the indicative is illustrated in the following examples...: "Les alegra que estudies / *estudias tanto". "Le avergonzaba que lo besara / *besaba en púbico". "Me alegro de que hayas / *has venido". It is quite apparent that a subjunctive form is obligatory in the embedded clause of each structure given.

Lo mismo afirma Kleiman (1974, p. 194): "Emotive verbs must govern a subjunctive verb... all emotives, whether they are factive or non-factive, govern a subjunctive complement”. Bosque (1990, p. 42) señala la universalidad de tal opinión entre los lingüistas:

${ }^{1}$ Se trata de lo que Terrell, y Hooper (1974, p. 488) denominan "comentario" (comment): "Since we have discovered no syntactic processes which critically depend on the semantic difference between mental states of emotion and value judgement, we will subsume both under the heading «comment»". 
"Las nociones semánticas que siempre se han utilizado para clasificar a los núcleos que seleccionan subjuntivo no son muy diferentes unas de otras. Entre ellas están... apreciaciones valorativas y afectivas".

Sin embargo, algunos lingüistas han notado que aparecen a veces cláusulas regidas por expresiones emotivas y valorativas en las que se usa el indicativo. Cook (1975, p. 118), por ejemplo, dice lo siguiente:

Although some grammarians and linguists do not concur, many agree... that the subjunctive is required after... "expressions of emotion"... Common usage, however, provides ample data to the contrary:

"Se alarma de que sucede eso" (Trinidad Estupinián, El Salvador). "Lamento que ha sucedido eso" (Sara Averbuj, Argentina).

"Temo que me han visto" (Carlos Salazar, El Salvador).

Anadón (1979, p. 44), en una encuesta en que participaron 245 estudiantes universitarios de cinco países hispanos (Panamá, Colombia, Ecuador, Perú, Chile), encuentra que $49 \%$ de los informantes admiten la oración "Sintió mucho que nadie postulaba al cargo" como una oración "completamente aceptable" (13\%) o "no completamente aceptable, pero se usa" (36\%).

Terrell y Hooper (1974, p. 488) también cuestionan la idea de que ciertas nociones semánticas siempre determinan el modo que se emplea en cláusula regida por ellas:

We have not been able to associate the choice of mood consistently with a certain semantic notion... We have identified an area of instability in the mood system... It is common for some speakers to use indicative in all types of presupposed complements. Thus either form would be accepted [in the following]: Me sorprendio que \{vino/viniera\}. Es bueno que Ud. \{llega/llegue\} a tiempo.

El presente estudio corrobora esto. He aquí algunos ejemplos extraídos del corpus²:

${ }^{2}$ Las citas de los corpora se identifican por medio de una abreviación del nombre de la ciudad, seguida de los números de la muestra y de la página, así que "воG-12: 162", por ejemplo, indica que la cita es del corpus de Bogotá, muestra 12, página 162. Estas son las siglas usadas: $\mathrm{BOG}=$ Bogotá, $\mathrm{BA}=$ Buenos Aires, $\mathrm{CAR}=$ Caracas, $\mathrm{HAB}=\mathrm{La}$ Habana, $\mathrm{LAP}=\mathrm{La}$ Paz, $\mathrm{LIM}=\mathrm{Lima}, \mathrm{MAD}=$ Madrid, $\mathrm{MEX}=$ México, SJO = San José de Costa Rica, sJN = San Juan de Puerto Rico, SNT = Santiago de Chile, $\operatorname{sev}=$ Sevilla. 
A mí personalmente me interesa mucho que si ha habido cosas positivas (воG-12: 162).

Se admira que a mi no se me olvida nada (воG-38: 517).

Cuando salí me sorprendió que vi frente a la estación de ferrocarril cinco hoteles inmensos (CAR-5: 82).

Cuando yo llego, oye, me parece raro que este hombre baja y dice, "Mire..." (CAR-17: 314).

Me agrada que esa tesis ha sido comentada por N.N. (LIM-18: 242).

Me repugna así francamente que la gente es mal agradecida (SANT4: 81).

Ha venido un profesor de Puerto Rico y se asombra de que todo el mundo pues tiene un "ticket" (MAD-13: 225).

Los comentarios que se han hecho sobre el empleo del indicativo en oraciones de este tipo son pocos y muy limitados, y no existe, que yo sepa, ningún estudio global dedicado exclusivamente a este fenómeno. Kany (1951) no dice nada al respecto, y Lope Blanch (1958, p. 383) lo describe como un "fenómeno que no ha despertado el interés de los investigadores". Aquí presento una investigación de esta construcción, en la que resumo los comentarios que se han hecho al respecto y describo su ocurrencia en el español hablado culto contemporáneo.

La base de datos para este estudio proviene de muestras de habla culta de doce ciudades, las cuales fueron recolectadas en entrevistas grabadas desde fines de la década de los sesenta hasta principios de los años ochenta. Las grabaciones luego fueron transcritas y publicadas, y para la presente investigación se han extraído todos los casos de uso del indicativo y subjuntivo, con treinta y cinco expresiones de reacción personal que se presentan en los corpora de las doce ciudades: Bogotá, Buenos Aires, Caracas, La Habana, La Paz, Lima, Madrid, México, San José de Costa Rica, San Juan de Puerto Rico, Santiago de Chile y Sevilla.

Un aspecto importante de estos materiales es su uniformidad, ya que representan muestras de habla recolectadas según normas preestablecidas. Los informantes de cada ciudad son hombres y mujeres en una distribución proporcional del $50 \%$. Constituyen tres generaciones, de acuerdo con la siguiente distribución y proporción: de 25 a 35 años de edad $=30 \%$; de 36 a 55 años $=45 \%$; y de más de 55 años $=25 \%$. Todos los informantes son nacidos o residentes, al menos durante las tres cuartas partes de su vida, en la ciudad objeto de estudio desde los cinco años. Son hijos de hispanohablantes, preferentemente nacidos en la misma localidad, y 
han recibido su instrucción primaria, secundaria y universitaria (o equivalente) en ella. Se han hecho cuatro tipos de grabaciones: diálogo dirigido entre uno o dos informantes y el investigador; diálogo libre entre dos informantes; grabación secreta de un diálogo espontáneo, y elocuciones en actitudes formales (clases, conferencias, discursos, etcétera) ${ }^{3}$.

\section{DiSTRIBUCIÓN GEOGRÁFICA DE LAS EXPRESIONES} DE REACCIÓN PERSONAL CON INDICATIVO

La frecuencia de empleo del indicativo con expresiones de reacción personal varía en las doce ciudades representadas en la base de datos, pero ocurre por lo menos una vez en todas las ciudades. No se trata, entonces, de un fenómeno puramente regional. La distribución geográfica del empleo del indicativo varía desde $33 \%$ para Sevilla, hasta $75 \%$ para Lima. Esta gran discrepancia entre una ciudad peninsular y una hispanoamericana sugiere la posibilidad de que tal uso sea más americano que peninsular. De hecho, Lope Blanch (1958, p. 383), lingüista español radicado en México, señala el empleo del indicativo en cláusula regida por verbo de emoción en el español de México, en particular, y de Hispanoamérica en general:

En el español de México no es raro... encontrar expresiones de estas clases construidas con verbo subordinado en indicativo...

${ }^{3}$ Los materiales que constituyen el corpus del presente estudio forman parte del "Proyecto de estudio coordinado de la norma lingüística culta de las principales ciudades de Iberoamérica y de la Península Ibérica". Un breve resumen del "Proyecto" se da en DeMello (1994b). Para una historia pormenorizada véase Lope Blanch (1986). Los tomos que se han completado hasta el momento son los siguientes, enumerados por orden alfabético según los nombres de las ciudades: Bogotá: Otálora de Fernández, y A. González 1986; Buenos Aires: Barrenechea 1987; Caracas: Rosenblat, y Bentivoglio 1979; La Paz: Marrone 1992; Lima: Caravedo 1989; Madrid: Quilis, Esgueva, y Cantarero 1981; México: Lope Blanch 1971, 1976; San José: Solano (manuscrito inédito, ca. 1994); San Juan: Morales, y Vaquero 1990; Santiago: Rabanales, y Contreras 1979, 1990; Sevilla: LamíQuiz, y PINEDA 1983. Los materiales de La Habana los constituyen treinta encuestas que me facilitó el profesor Joseph H. Matluck de la Universidad de Texas, en Austin, en forma de manuscrito inédito. El único tomo de habla inculta que se ha publicado hasta la fecha es el de México (Lope Blanch 1976) y, a excepción de algunos casos especiales indicados en los lugares pertinentes, estos materiales no se incluyen en los porcentajes que se refieren a la ciudad de México en el presente trabajo. 
Seguramente también en otros países de América podrán hallarse construcciones semejantes... Recojo algunos ejemplos mexicanos con verbos de emoción: "Estoy muy satisfecha de que supo terminarlo él solo"; "Tengo miedo de que tu hermano fue el que se lo dio"; "No lo puedo remediar: me da coraje que lo hizo sin mi permiso".

Sin embargo, Lope Blanch (p. 384) reconoce que este fenómeno también existe en el español peninsular: "Es indudable que no se trata de un fenómeno exclusivamente americano; pueden hallarse ejemplos similares en España: «Mucho me alegra que no ha caído en el vacío mi escrito» $(A B C, 1955)$ ".

El empleo peninsular de tal construcción en el español parece no ser un fenómeno moderno, puesto que ya se encuentra en documentos muy antiguos. Tarr (1922, pp. 137, 160), por ejemplo, cita casos como éstos:

Muy alegre quedo, señora mía, que se ha ofrecido caso en que conozcas lo que yo sé hacer (La Celestina).

Mandó dar pregón... quel gradeçiessen de que no los mandava matar (Crónica general).

A ti he de gradeçer por que so aún viva (Crónica general).

Keniston (1937, § 28.26) da numerosos ejemplos en escritores del siglo XVI, entre ellos los siguientes:

"Pésame que lo sujetas o cosas mortales".

"Me plaze que te sonrríes".

"Me rezelava que nos lo havían de meter en casa".

"Havemos miedo que nos apedreará las viñas".

Sin embargo, en lo que se refiere al español peninsular contemporáneo, Bosque (1990, p. 46) señala que esta construcción es de empleo muy limitado, y que ocurre mayormente en Hispanoamérica: "La pauta de (47) [Les reprocho que no me \{hacen/hagan\} caso] está muy limitada en el español peninsular, mientras que en el español americano es mucho más productiva”. En efecto, el presente estudio confirma esto. Una comparación entre las dos ciudades españolas, Madrid y Sevilla, y las diez ciudades hispanoamericanas revela una preferencia por el indicativo que es mayor en el uso hispanoamericano $(50 \%)$ que en el peninsular $(33 \%)$. 
Los casos de indicativo vs. los de subjuntivo para las doce ciudades se dan en el Cuadro $1^{4}$.

CuAdro 1

Distribución geográfica de casos de indicativo/subjuntivo con expresiones de reacción personal

\begin{tabular}{lrr}
\hline Ciudad & Indicativo & Subjuntivo \\
\hline Bogotá & $16(53 \%)^{5}$ & $14(47 \%)$ \\
Buenos Aires & $8(67 \%)$ & $4(33 \%)$ \\
Caracas & $12(60 \%)$ & $8(40 \%)$ \\
La Habana & $2(40 \%)$ & $3(60 \%)$ \\
La Paz & $6(46 \%)$ & $7(54 \%)$ \\
Lima & $3(75 \%)$ & $1(25 \%)$ \\
Madrid & $3(30 \%)$ & $7(70 \%)$ \\
México & $8(40 \%)$ & $12(60 \%)$ \\
San José & $9(56 \%)$ & $7(44 \%)$ \\
San Juan & $10(43 \%)$ & $13(57 \%)$ \\
Santiago & $21(45 \%)$ & $26(55 \%)$ \\
Sevilla & $2(40 \%)$ & $3(60 \%)$ \\
Total & $100(49 \%)$ & $105(51 \%)$ \\
\hline
\end{tabular}

Debe advertirse que en los recuentos del Cuadro 1 se han incluido solamente las expresiones que ocurren por lo menos una vez con indicativo; son las que se dan en el Cuadro 2.

${ }^{4}$ Se debería tomar en cuenta, al consultar las cifras de distribución dadas en los cuadros, que la extensión de los corpora de las doce ciudades difiere entre sí, a veces de manera muy notable. El corpus de Santiago, por ejemplo, es casi seis veces más grande que el de Sevilla. A continuación se dan los tamaños de los corpora en términos de 10 kilobytes, redondeados a los 100 bytes más próximos: Bogotá, 147.71; Buenos Aires, 159.33; Caracas, 164.66; La Habana, 103.22; La Paz, 121.86; Lima, 80.59; Madrid, 85.50; México, 105.68; San José, 90.78; San Juan, 101.58; Santiago, 262.45 y Sevilla, 45.57.

${ }^{5}$ Los porcentajes en este trabajo han sido redondeados. Si el decimal es de .5 o más, se aproxima al decimal siguiente, así, por ejemplo, $67.5 \%=68 \%$, $67.4 \%=67 \%$, etc. Las únicas excepciones las constituyen los casos en que dos porcentajes contrastivos tienen un decimal de .5 , en cuyo caso va indicado el decimal. 
Cuadro 2

Distribución de casos de indicativo/subjuntivo con expresiones de reacción personal

\begin{tabular}{lrrlrr}
\hline Expresión & Ind. & Sub. & Expresión & Ind. & Sub. \\
\hline Admirar & 3 & 2 & Interesante & 3 & 6 \\
Afán & 1 & 2 & Interesar & 3 & 3 \\
Agradar & 1 & 1 & Lamentar & 1 & 1 \\
Agradecer & 1 & 7 & Lástima & 4 & 5 \\
Angustia & 6 & 0 & Lógico & 5 & 11 \\
Ansia & 1 & 0 & Natural & 1 & 9 \\
Asombrar & 3 & 1 & Perdonar & 1 & 12 \\
Asustar & 2 & 2 & Ridículo & 1 & 0 \\
Bueno & 1 & 13 & Pena & 3 & 7 \\
Chasco & 1 & 0 & Quejarse & 15 & 0 \\
Contento & 1 & 2 & Raro & 1 & 5 \\
Criticar & 5 & 3 & Repugnar & 1 & 0 \\
Curioso & 5 & 1 & Satisfacción & 5 & 1 \\
Decepcionar & 1 & 0 & Sorprender & 3 & 3 \\
Desgracia & 3 & 2 & Sorpresa & 11 & 0 \\
Emoción & 1 & 0 & Temer & 2 & 6 \\
Extraño & 2 & 0 & Tristeza & 1 & 0 \\
Inquietud & 1 & 0 & & & \\
& & & Total & 100 & 105 \\
\hline
\end{tabular}

Existe la posibilidad de que no todas las expresiones de reacción personal con indicativo que ocurren en el corpus estén incluidas en el Cuadro 2, puesto que la lista se limita forzosamente a expresiones notadas por mí al leer los materiales, y a las que he buscado deliberadamente. Esta búsqueda incluye, además de todas las expresiones que se me ha ocurrido rastrear, las que se encuentran mencionadas en las obras lingüísticas consultadas ${ }^{6}$. La lista completa se compone de 113 expresiones de reacción personal, 35 de las cuales se dan en el Cuadro 2. Las otras 78 expresiones examinadas, pero no incluidas en el Cuadro 2 por no regir indicativo en el corpus, son las siguientes (el número de casos del subjuntivo en cláusula subordinada a la expresión se da entre

${ }^{6}$ Para este fin fue de especial ayuda la lista que se encuentra en KLEIN (1974, pp. 140-141), la cual se compone de 76 "verbos factivos", o sea, verbos emotivos/evaluativos, que normativamente rigen el subjuntivo. 
paréntesis): aborrecer (0); aburrir (1); agradable (1); aguantar (1); alarmar (0); alegrar (5); apenar (0); apetecer (2); asco (1); atraer (0); azorar (1); bien (20); celebrar (1); complacer (2); confundir (0); decepción (0); defender $(0)$; denunciar (0); deplorar $(0)$; desesperarse (1); desilusionar $(0)$; despreocuparse $(0)$; dichoso $(0)$; disgustar $(0)$; divertir $(0)$; doler (4); depresión (0); deprimir $(0)$; encantar (12); enfermar $(0)$; enojar $(0)$; entristecer $(0)$; entusiasmar $(0)$; escandalizar $(1)$; excitar (0); extraordinario (0); fascinar (2); fastidiar (0); felicidad (1); feliz (1); gustar (48); horrorizar (2); implorar (1); importar (26); indignante (1); indignarse (0), justificar (6); locura (0); mal (3); malo (3); maravillar (0); mejor (11); miedo (21); milagro (0); molestar (7); odio (1); orgulloso (1); peor (0); pesar (0); placer $(0)$; preocupar (8); protestar $^{7}(0)$; rabia $(1)$; razonable $(0)$; resentir $(0)$; respetar $(0)$; reventar $(0)$; significativo $(0)$; temor $(7)$; terror (3); tolerar $(0)$; tragedia $(0)$; tremendo (0); triste (3); útil (2); valer la pena (9); vergonzoso (2); vergüenza (3). Si se incluyeran estos casos del empleo del subjuntivo en los 105 ya contados, el total de casos de subjuntivo con expresiones de reacción personal sería de 331, en contraste con sólo 100 de indicativo. De esta forma, el empleo del indicativo con tales expresiones sería $23 \%$ y el del subjuntivo, $77 \%$. La frecuencia del indicativo, aunque mucho menor que el $49 \%$ que se arroja cuando se limitan los recuentos a las expresiones que ocurren en el corpus con ambos modos, es, de todas maneras, bastante significativa.

CON VERbo SUbORdinAdo EN EL PERFECTO ACTUAL

Según Vogel (1979, p. 88) no es posible emplear el indicativo en cláusula subordinada a una expresión de emoción si el tiempo verbal de tal cláusula es el perfecto actual. De ahí que hablantes que aceptan oraciones como "Les alegra que vienes", rechazarían oraciones como "Les alegra que *has venido": "If we explore data

${ }^{7}$ Según Butt, y Benjamin (1994, § 16.6.2), "protestar de que" rige el indicativo. En mi corpus sólo ocurre el verbo "protestar" o el sustantivo "protesta" con "porque", siempre en indicativo. Son los siguientes casos: "A través de sus protestas porque se acabó la moral, están expresando es sus deseos fallidos" (BOG-11: 154); "Al teatro se iba con sombrero... pero la gente de atrás no protestaba porque no les dejaban ver" (MAD-15: 247); "Luego los yaquis protestan porque les quitan unas tierras" (MEX-29: 393); "Los muchachos empiezan... a protestar porque no empieza el espectáculo" (sJN-3: 68); "Cuatro o cinco niños contestatarios protestan porque no saben" (SEv-21: 258). 
expressing emotion, we find that the indicative may be possible for some speakers... However, the indicative is no longer possible if the embedded verb is present perfect: «A tus padres les alegra que \{hayas/*has\} estudiado tanto»".

El presente estudio no ratifica esto. En la base de datos se presentan 22 ejemplos en que el verbo de la cláusula subordinada a una expresión de reacción personal va en perfecto actual, y de éstos, 6 (27\%) son casos de indicativo. Se trata de los siguientes:

Me agrada que esa tesis ha sido comentada por N.N. (LIM-18: 243). Es curioso que ella ha estudiado en el Colegio Alemán y ha ido a los Estados Unidos y tú has estudiado en el Americano y has ido a Alemania (LAP-22: 228).

Es curioso que en un país tan chico han avanzado en eso (SANT46: 354).

Es extraño que yo un pintor básicamente realista antiguo todos mis estudiantes han salido pintores abstractos (sJN-2: 51).

A mí personalmente me interesa mucho que si ha habido cosas positivas (воG-12: 162).

Y el pueblo se queja tremendamente de que los militares han querido implantar la igualdad social (BOG-25: 331).

Aunque en tales casos la frecuencia del indicativo (27\%) es mucho menor que la del subjuntivo $(73 \%)$, no es de ninguna manera despreciable.

EXPRESIONES DE REACCIÓN PERSONAL CON REFERENCIA A PARTES DE LA ORACIÓN

Como se ve en el Cuadro 2, las expresiones de reacción personal que aparecen en la base de datos son de tres tipos (en lo que a partes de la oración se refiere): sustantivo, adjetivo y verbo. Con sustantivo, el empleo del indicativo sobrepasa más de dos veces el del subjuntivo, mientras que con adjetivo es a la inversa, o sea que el uso del subjuntivo es más de dos veces más frecuente que el del indicativo. Con los verbos, el empleo de los dos modos es de igual frecuencia (Cuadro 3). 
Cuadro 3

Distribución de casos de indicativo/subjuntivo con partes de la oración

\begin{tabular}{lrr}
\hline Parte & Indicativo & Subjuntivo \\
\hline Sustantivo & $38(69 \%)$ & $17(31 \%)$ \\
Adjetivo & $20(30 \%)$ & $47(70 \%)$ \\
Verbo & $42(51 \%)$ & $41(49 \%)$ \\
Total & $100(49 \%)$ & $105(51 \%)$ \\
\hline
\end{tabular}

El hecho de que la frecuencia del indicativo sea marcadamente más alta con sustantivos que con adjetivos o verbos, posiblemente se deba a que una cláusula que modifica a un sustantivo muchas veces va en aposición al sustantivo en vez de estar subordinada a éste, de ahí que no exista la subordinación concomitante al empleo del subjuntivo. De esto se hablará con más detalle después.

\section{EXPRESIÓN DE SORPRESA}

Es de interés notar que los casos del empleo del indicativo con expresiones de sorpresa, representados por admirar, asombrar, sorprender y sorpresa, suman veinte, forman así casi la cuarta parte (23\%) de los 87 casos de expresiones de reacción personal con indicativo. Si se consideran todas las expresiones de sorpresa, se ve que en total hay veinte casos de indicativo $(77 \%)$, en contraste con sólo seis de subjuntivo (23\%). Estos son algunos ejemplos:

Se admira que a mí no se me olvida nada, ¿no?, nada, nada (BOG38: 517).

Repito, mis hijos se admiran un poco de que yo conozca eso (CAR5: 75).

Vino un norteamericano, y estaba admirado de que los cubanos le rindiéramos homenaje a Washington (нАВ-37: 668).

$\mathrm{Al}$ pedir un certificado de estudios me he encontrado muy admirado de que las notas no eran tan malas como yo creía (LIM12: 172).

Ha venido un profesor de Puerto Rico y se asombra de que todo el mundo pues tiene un "ticket" (MAD-13: 225).

Estoy así asombrado de que nos haya salido tan bien (LAP-13: 136).

Me parece sorprendente que el Instituto Caro y Cuervo esté haciendo una encuesta sobre el uso del lenguaje bogotano (BOG37: 500). 
Cuando salí me sorprendió que vi frente a la estación de ferrocarril cinco hoteles inmensos y magníficos (CAR-5: 82).

Tuve la más agradable sorpresa de que Chile se conocía en los círculos más cultos (SANT-51: 430).

De pronto se lleva uno la sorpresa de que el carro está intacto (BOG-27: 370).

El número de casos de indicativo $(I)$ y subjuntivo $(S)$ para cada expresión de sorpresa es el siguiente: admirar: I: 3, S: 2; asombrar: I: 3, S: 2; sorprender. I: 3, S: 3; sorpresa: I: 11, S: 0. Como se ve, todas las cláusulas que modifican el sustantivo "sorpresa", once en total, van con el verbo en indicativo. Tal hecho corrobora lo que se ha dicho en el apartado anterior referente al empleo relativamente alto del indicativo en cláusula apositiva con antecedente sustantival. Esta situación conduce a una menor independencia semántica entre la cláusula subordinada y su antecedente y resulta en una cláusula que explica el sustantivo que modifica, en lugar de una cláusula que vaya subordinada a tal sustantivo. Cuando discuta la independencia semántica que puede conducir al empleo del indicativo, abundaré en esta cuestión.

EXPRESIÓN EMOTIVA SEGUIDA POR "DE QUE"

Según Butt y Benjamin (1988, § 16.2.2.i), el verbo emotivo seguido por "de que" a veces rige el indicativo, sobre todo en lenguaje popular:

After expressions denoting emotional reactions which are followed by de que the subjunctive is more normal in formal language and safer for non-natives, although the indicative tenses are sometimes found in informal speech when the verb is in the present or past: $M e$ alegré de que pensaban / pensaran hacerlo. Se indignaba de que sus suegros (creian) / creyeran en la pena de muerte. Se horrorizaba de que la (trataban) /trataran así.

Es interesante advertir que en tres de estos ejemplos se trata de un verbo pronominal seguido de preposición ("alegrarse de", "indignarse de", "horrorizarse de"), se confirma así una observación hecha por Porto Dapena (1991, pp. 107-109) de que algunos de los verbos "afectivos", que normativamente exigen subjuntivo, también admiten el indicativo cuando van seguidos de complemento preposicional: 
Una clase de verbos que exigen siempre subjuntivo son los llamados "afectivos"... Con oración sustantiva como complemento preposicional: alegrarse de, arrepentirse de, contentarse de, dolerse de, jactarse de, lamentarse de, maravillarse de, sorprenderse de... algunos de estos verbos... admiten fácilmente indicativo sin cambio de significado: así preocuparse, lamentarse de, quejarse de.

En la segunda edición de su libro (1994, § 16.6.2), Butt y Benjamin añaden que, aunque se emplean los dos modos con "lamentarse de", según sea o no un verbo de emoción, solamente se usa el subjuntivo con "lamentar": "Lamentar «to regret the fact that» takes the subjunctive. Lamentarse de que «to lament the fact that» takes the subjunctive when it expresses an emotional reaction and the indicative when it merely makes a statement". No obstante, en mi corpus se encuentran dos casos de cláusulas subordinadas al verbo "lamentar": en uno rige el indicativo mientras que en el otro rige el subjuntivo; parece, pues, que sí puede ocurrir el indicativo con "lamentar":

Los israelitas lamentan que se haya muerto (BA-25: 239).

Ella no lamenta en absoluto —-seguramente, qué sé yo, este matrimonio está totalmente roto- no; no lamenta eso, sino que lamenta el que el colchón ya no tenía ni media pluma (SANT-12: 207).

También se debería recordar que Cook (1975, p. 118), como indiqué antes, incluye un caso de "lamentar" entre sus ejemplos de verbos de emoción que pueden regir el indicativo: "Lamento que ha sucedido eso" (Sara Averbuj, Argentina). Sin embargo, en las oraciones que componen el corpus del presente estudio se confirma el empleo más frecuente del indicativo con "de que" que con "que". En el corpus, las expresiones que se construyen con "que" arrojan un porcentaje de $39 \%$ para el uso del indicativo, en contraste con $61 \%$ para el del subjuntivo; mientras que, para las expresiones que se construyen con "de que", estos porcentajes son de 80 para el indicativo y sólo 20 para el subjuntivo. Así que se favorece el empleo del indicativo sobre el subjuntivo dos veces más en una cláusula encabezada por "de que", que en una cláusula encabezada por "que" (Cuadro 4). 
Cuadro 4

Indicativo vs. Subjuntivo con "que" y "de que"

\begin{tabular}{lrr}
\hline & Indicativo & Subjuntivo \\
\hline Que & $61(39 \%)$ & $95(61 \%)$ \\
De que & $39(80 \%)$ & $10(20 \%)$ \\
Total & $100(55 \%)$ & $105(45 \%)$ \\
\hline
\end{tabular}

\section{EXPRESIÓN EMOTIVA QUE PRESENTA INFORMACIÓN}

Cook (1975, p. 161) nota que una de sus informantes explicó la posibilidad de empleo del indicativo con el verbo "gustar" en una cláusula informativa: "An Argentine informant... explained that if she were informing a friend of her reaction to a certain experienced event, she would say: No me gusta que mi marido usa esa corbata".

Borrego, Asencio y Prieto (1985, pp. 35-36) señalan que el empleo del indicativo en cláusula regida por verbo afectivo a veces se debe al hecho de que tal verbo es también de comunicación y que imparte información:

Tanto [los predicados de valoración] como los de sentimiento en ocasiones no se limitan a recoger un hecho que ya se supone conocido para comentarlo: junto al comentario o valoración se incluye la información de que lo que se dice en la subordinada ha sucedido, sucede o va a suceder. En este uso "llevan dentro" un verbo de comunicación y, en consecuencia, aparecen en indicativo los verbos que de ellos dependen.

Bosque (1990, p. 46) concuerda con esto: "Creemos que es correcta la observación que hacen estos autores cuando apuntan que esos verbos de sentimiento o juicio pueden «funcionar a la vez como verbos de comunicación»". Lo mismo dice Porto Dapena (1991, p. 109): "En algunos de estos verbos puede predominar la idea de comunicación o información, por lo que admiten fácilmente indicativo sin cambio de significado; así preocuparse, lamentarse de, quejarse de".

Oviedo (1974, pp. 76-78) hace uso del factor [ \pm ENFOQUE] para describir la alternancia modal en oraciones como "Me alegro de que conseguiste/hubieras conseguido empleo”, pero sus comentarios al respecto revelan que en realidad se trata de la intensidad del valor informativo del evento subordinado al verbo matriz: 
"The indicative form calls attention to the presupposed event, as if it constituted the heart of the message; and the subjunctive form expresses the event as a secondary message unit, as an unimportant event".

En relación con esto, Lavandera (1983, p. 230) explica que el subjuntivo es el modo que se emplea cuando el hablante no quiere enfocar la atención del intelocutor en lo enunciado: "Shifts to utterances with the subjunctive mood take place when the speaker switches to issues that he neither wants to leave unmentioned nor wants to make the center of the discussion".

Lope Blanch (1958, p. 384) también señala la importancia del valor informativo del verbo de emoción que rige una cláusula con el verbo en indicativo, y apunta que la afirmación del hecho se sobrepone al contenido emotivo: "Se produce sólo cuando se trata de acciones pretéritas o presentes, es decir de hechos ciertos cuya realidad se afirma". Guitart $(1982,1984,1987)$ encuentra que cuanto más informativo sea el contenido de una cláusula, más común será el empleo del indicativo en ella, y que esto ocurre aunque la cláusula vaya regida por una expresión que normativamente rige el subjuntivo, como, por ejemplo, la expresión de reacción personal: "If the speaker rates the clause as information he will use the Indicative, except if he is commenting on information which he adjudges to be either shared by the hearer or not unexpected if unshared" (1984, p. 165).

Por mi parte, en otro lugar (DeMello 1990, pp. 292-303, 319327) he presentado un análisis del contraste indicativo/subjuntivo también basado en nociones de información = indicativo y comentario = subjuntivo: "A speaker may relate an event in one of two ways: (1) he may treat the event as a piece of information and use the indicative... or (2) he may make a commentary on this information and conceptualize the event by placing the verb in the subjunctive”. Según este análisis, los verbos emotivos son comentarios sobre el evento que se presenta en la cláusula subordinada; el comentario es la parte informativa de la oración y por eso va en indicativo, mientras que el evento en la cláusula subordinada es algo ya consabido y por eso el verbo de esta cláusula va en subjuntivo. Sin embargo, a veces, aun con verbos emotivos, el hablante presenta el evento en la cláusula subordinada como información y no como algo ya consabido, y en tal caso esto se refleja en el empleo del indicativo en la cláusula subordinada. De ahí que sea evidente que lo que conduce al empleo del indicativo o subjuntivo no es el verbo matriz en sí, sino el valor semántico que el 
hablante atribuye a este verbo. Klein (1975, p. 353) ha notado esto: "It is the meaning of the main verb that calls for assertion of the occurrence of the complement... this explanation captures a generalization about... the verbs of reporting and claiming... which explains their occurrence with complements in the indicative". Todo esto indica que el contraste modal indicativo/subjuntivo es más sensible al contexto de lo que se había creído. Cook (1975, pp. 164-165) también lo señala: "The evidence contradicts Hadlich's assertion that «the subjunctive forms do not mean anything in themselves...»...The sophisticated speakers encode nuances that are independent of the surface structures of the sentence". Blake (1985, p. 171) concuerda: "The exact meaning of any particular use [of the subjunctive] can only be determined with reference to the surrounding context; in other words, the subjunctive is context-sensitive".

Blake advierte que en entrevistas efectuadas en San Luis Potosí, México, en las que la investigadora era una persona no nativa del lugar - con lo que se creaba un ambiente lingüístico en que los informantes daban noticias a la interlocutora- el empleo modal resultó contrario a lo que se esperaba. El indicativo se utilizó a veces en situaciones en que normativamente se emplea el subjuntivo, a saber, en cláusulas regidas por expresiones emotivas (como "tener miedo", "sorprender", etc.) o evaluativas ("ser justo", "parecer bueno", etc.). Estos son algunos de los ejemplos citados por Blake (1985, pp. 168-169; 1987, pp. 353-356):

"Tenía miedo de qué iba a pasar".

"Me sorprende que no tienen prisa para nada".

"Me da coraje que no tienen interés por estudiar".

"No es justo que no les cuesta la escuela".

"Me parece bueno que tenemos buenos colegios".

"A los niños les gusta que los llevan a nadar".

En todos estos ejemplos, el hablante está informando al interlocutor de algo que éste no sabe, de manera que los verbos, básicamente de tipo emotivo, se utilizan en sentido de verbos comunicativos.

El CASO DEL Verbo “QUeJARSE"

Un buen ejemplo de un verbo en que el valor comunicativo-informativo se sobrepone al valor emotivo es "quejarse", verbo que, 
aunque representa una emoción, lleva dentro la idea de proporcionar información al interlocutor, aunque sea en forma de queja. Borrego, Asencio y Prieto (1985, p. 36) explican la alternancia modal con este verbo de la siguiente manera:

Me quejo de que mi hijo estudia poco. (Valoro, pero a la vez informo.) Me quejo de que mi hijo estudie poco. (Presento el carácter poco estudioso de mi hijo como algo conocido y doy el carácter de información principal al efecto psicológico de ello: fundamentalmente valoro.)

En cambio, Butt y Benjamin (1994, § 16.6.2) afirman que el verbo de una cláusula subordinada al verbo quejarse siempre está en indicativo, puesto que este verbo se emplea para enunciar algo, no para expresar una emoción: "Quejarse de que «to complain that..." seems to foreign speakers to be an emotional reaction, but it is in fact treated as verb of statement and is always followed by the indicative".

El empleo indicativo/subjuntivo en cláusula regida por "quejarse" tal como se encuentra en mi corpus confirma esto, puesto que, como ya se ha señalado antes (véase Cuadro 2), los quince casos de cláusula regida por "quejarse" tienen el verbo en indicativo; he aquí algunos ejemplos:

El pueblo se queja tremendamente de que los militares han querido implantar la igualdad social (BОG-25: 331).

Me quejo de que yo tengo que trabajar (BА-21: 20).

Ayer uno de los senadores se quejó de que había una especie de mercado de fariseos (CAR-16: 280).

Ellos se quejan de que no voy a las reuniones (НАв-37: 668).

Se quejaban de que a la mujer le pagan menos (LAP-12: 125).

Nos quejamos de que hay muchas industrias de extranjeros (MEX29: 398).

Ellos siempre se quejaban de que nunca los acogieron bien (SANT25: 406).

\section{LAS EXPRESIONES DE SORPRESA}

La expresión de sorpresa es otra que demuestra un alto porcentaje de oraciones subordinadas con verbo en indicativo. Si se estudian estos casos, se ve que el empleo del indicativo se debe a que el 
evento que causa la sorpresa es presentado por el hablante como información. Estos son algunos ejemplos:

Uno se sorprende que ve que hay muchachos que dan diez materias en un año (BA-1: 34).

Cuando salí me sorprendió que vi frente a la estación de ferrocarril cinco hoteles inmensos y magníficos (CAR-5: 82).

Me sorprendió muchísimo que... me tocó enseñar Moneda y Banca (sNJ-9: 182).

En cambio, cuando el hablante se refiere a eventos ya sabidos por el interlocutor, utiliza el subjuntivo, puesto que ya no se trata de dar información en la cláusula subordinada. Un ejemplo de esto es la siguiente oración en la que el hablante se refiere a la encuesta que le están haciendo. En este caso, la parte informativa de la oración se limita a la expresión de sorpresa, la cual se enuncia en indicativo, y la causa de tal sorpresa es algo consabido que, por eso, se formula en subjuntivo:

Me parece sorprendente que el Instituto Caro y Cuervo esté haciendo una encuesta particularmente sobre el uso del lenguaje bogotano (BOG-37: 500).

\section{El VERBo “AGRADECER"}

El verbo “agradecer", en cambio, es por su naturaleza semántica un verbo que normalmente rige una cláusula en la que el evento es algo conocido por el interlocutor, ya que el agradecimiento se le expresa a alguien por algo que obviamente conoce la persona a quien va dirigido. Siendo así, se esperaría que la cláusula subordinada a "agradecer" llevara el verbo en subjuntivo. En efecto, tal es el caso en casi todos los ejemplos de este verbo que se encuentran en la base de datos. De los ocho casos de cláusula subordinada al verbo "agradecer", siete llevan el verbo en subjuntivo; estos son algunos ejemplos:

Le agradezco muchísimo que usted coopere con nosotros en este estudio (MEX-5: 67).

Yo te agradezco que me hayas dado un poquito de tu tiempo (SNT2: 51$)$.

Y te agradezco que sacaras el rato porque yo sé que tenés un horario demasiado estricto (sjo-4). 
El único ejemplo del empleo del indicativo con "agradecer" lo representa un caso en que el hablante imparte información en la cláusula subordinada a este verbo:

Entonces me dijo: "Mire, agradezca usted que está aquí uno de los principales dirigentes" (LAP-16: 167).

En esta oración, la información encerrada en "está aquí uno de los principales dirigentes" es algo que el interlocutor no sabía.

\section{INDICATIVO/SUBJUNTIVO Y LA INDEPENDENCIA SEMÁNTICA}

Hemos visto que el empleo del indicativo con expresiones de reacción personal se encuentra mayormente en cláusulas regidas por "de que" y en cláusulas cuyo verbo proporciona información. Cabe preguntarse entonces, qué tendrán en común estos dos tipos de cláusulas, y la respuesta, a mi parecer, es que los dos comparten el rasgo de la independencia, o sea que representan situaciones lingüísticas en que la cláusula no va tan estrechamente subordinada al verbo matriz, como sería el caso de las cláusulas contrastivas en que el verbo va en subjuntivo. En otro ensayo (DeMello, 1994a), he expuesto la teoría de que el dequeísmo es cuestión de una selección estilística, basada en el grado de independencia semántica que el hablante quiera asignar a la cláusula encabezada por "que", de tal manera que una cláusula subordinada a "decir/creer de que", por ejemplo, es semánticamente más independiente del verbo matriz de lo que lo sería con una subordinada a "decir/ creer que". El empleo de la preposición "de" liga la cláusula a la expresión de reacción personal sin subordinarla a ésta. En forma parecida, la cláusula informativa - por eso no presupuesta- tampoco va subordinada semánticamente al verbo matriz, sino que funciona como su objeto directo, de ahí que lleve el verbo en indicativo. Al hablar aquí en términos de mayor o menor dependencia de la cláusula, es claro que no me refiero a nociones sintácticas, sino más bien a una dependencia de tipo semántico.

\section{Conclusiones}

El presente estudio permite llegar a ciertas conclusiones respecto al empleo del indicativo con expresiones de reacción personal en 
el habla culta. Lo primero que se nota es que este fenómeno no es de tipo regional, puesto que ocurre en las doce ciudades representadas en la base de datos. Sin embargo, los recuentos sí sugieren la posibilidad de que tal fenómeno sea más americano que español, puesto que la preferencia por el indicativo es sólo de $36 \%$ en el corpus peninsular, en comparación con $57 \%$ en el hispanoamericano.

Aunque se ha dicho que el empleo del indicativo en tal construcción no es posible si el tiempo del verbo subordinado es el perfecto actual, el corpus muestra que la frecuencia del indicativo (27\%) en tal caso, aunque mucho menor que la del subjuntivo (73\%), es significativa.

Por lo que respecta a las partes de la oración, el indicativo ocurre con más alta frecuencia en cláusula regida por sustantivo $(69 \%)$. También prevalece el indicativo en cláusula regida por verbo, aunque con menor frecuencia (58\%). En cambio, no es prevaleciente el empleo del indicativo en cláusula regida por adjetivo $(35.5 \%)$. Se sugiere aquí que esta alta frecuencia de indicativo en cláusula regida por sustantivo tal vez se deba a la relación de aposición que puede existir entre sustantivo y cláusula que lo modifica. Esto se ve en particular en el empleo del indicativo con expresiones de sorpresa, muy alto en general, que llega a ser total en las once cláusulas dependientes del sustantivo "sorpresa".

Algunos lingüistas han señalado cierta tendencia a utilizar el indicativo con el verbo emotivo seguido de cláusula encabezada por "de que". Esto se ratifica aquí, pues la frecuencia de indicativo es casi dos veces más alta en tal cláusula $(81 \%)$ que en la encabezada por "que" (44\%).

Varios lingüistas han señalado que el empleo del indicativo por el subjuntivo en cláusula subordinada a expresión de reacción personal se debe al valor informativo de esa cláusula. El presente estudio confirma esto, puesto que en gran parte de las oraciones en que se encuentra el verbo subordinado en indicativo, es evidente que el hablante está informando al intelocutor de algo que éste no sabe, o sea que el verbo matriz, aunque básicamente de tipo emotivo, se utiliza con el sentido de verbo comunicativo. Un buen ejemplo es el verbo "quejarse", que si bien representa una emoción, lleva dentro la idea de ofrecer información al interlocutor. En el corpus, los quince casos de cláusula regida por "quejarse" tienen el verbo en indicativo. La expresión de sorpresa es otra que muestra un alto porcentaje de oraciones subordinadas con verbo en indicativo, y en estos casos también, el evento que 
causa la sorpresa es expuesto por el hablante como información. En cambio, cuando el hablante se refiere a eventos ya sabidos por el interlocutor, la expresión de sorpresa rige una cláusula con verbo en subjuntivo. En casi todas las cláusulas subordinadas al verbo "agradecer" - que normalmente rige una cláusula en que el evento es algo conocido por el intelocutor- se encuentra el verbo en subjuntivo, tal como se esperaría. El único ejemplo del empleo del indicativo es un caso en que el hablante da información en la cláusula subordinada.

El empleo del indicativo con expresiones de reacción personal en cláusulas regidas por "de que" y en cláusulas cuyo verbo imparte información, conduce a pensar que el motivo para su uso en esos casos será cuestión de independencia; la cláusula no va tan estrechamente subordinada al verbo matriz, como sería el caso de aquella en que el verbo está en subjuntivo.

\author{
George DeMello \\ University of Iowa
}

\title{
BIBLIOGRAFIAA
}

Anadón Rojas, Silvia 1979. El subjuntivo en el español de Sudamérica. University of Michigan, Ann Arbor (tesis doctoral inédita ).

Barrenechea, Ana María (ed.) 1987. El habla culta de la ciudad de Buenos Aires. Materiales para su estudio. 2 ts. Universidad Nacional de Buenos Aires, Buenos Aires.

Blake, Robert 1985. "From research to the classroom: Notes on the subjunctive", $H, 68,166-173$.

_ 1987. "El uso del subjuntivo con cláusulas nominales: regla obligatoria o variable”, en Actas del I Congreso Internacional sobre el Español de América.

Eds. H. López Morales y M. Vaquero. Academia Puertorriqueña de la Lengua Española, San Juan, pp. 351-360.

Borrego, J., J. G. Asencio, y E. Prieto 1985. El subjuntivo. Valores y usos. Sociedad General Española de Librería, Madrid.

Bosque, IGNACio 1990. "Las bases gramaticales de la alternancia modal. Repaso y balance", en Indicativo y subjuntivo. Ed. I. Bosque. Taurus, Madrid, pp. 13-65.

Butt, John, \& Carmen Benjamin 1994. A new reference grammar of modern Spanish. Edward Arnold, London [1ํㅡㄹ ed.: 1988].

Caravedo, Rocío (ed.) 1989. El español de Lima. Materiales para el estudio del habla culta. Pontificia Universidad Católica del Perú, Lima.

Cook LeStrang, Gerard 1975. Mode, code, and performance: Analyses and discussions of the indicative/subjunctive contrast in Spanish with reference to a theory of language use. University of California (tesis doctoral inédita). 
DeMello, George 1994a. "El dequeísmo en el español hablado contemporáneo: un caso de independencia semántica”, $H L, 6$ (en prensa).

— 1990. Español contemporáneo. University Press of America, Lanham, MD.

— 1994b. "Some syntactic pecularities of Peninsular and New World Spanish as reflected in the Proyecto de Estudio de la Norma Lingüistica Culta", en Columbus quincentenary: Encounter and aftermath 1492-1992. University of Iowa, Iowa City, pp. 145-155.

Gili y Gaya, Samuel 1964. Curso superior de sintaxis española. Bibliograf, Barcelona.

Guitart, Jorge 1982. "On the use of the Spanish subjunctive among SpanishEnglish bilinguals”, Word, 33, 59-67.

— 1987. "Sobre el uso del subjuntivo español en dos dialectos caribeños: análisis pragmático”, BICC, 42, 141-148.

— 1984. "Syntax, semantics, and pragmatics of mood in Spanish noun clauses", HiJ, 6, 159-174.

Kany, Charles E. 1951. American Spanish syntax. University of Chicago Press, Chicago.

Keniston, Hayward 1937. The syntax of Castilian prose. University of Chicago Press, Chicago.

Kieiman Bustos, Angela 1974. A syntactic correlate of semantic and pragmatic relations: The subjunctive mood in Spanish. University of Illinois, Urbana-Champaign (tesis doctoral inédita).

KLeIN, Flora 1975. "Pragmatic constraints on distribution: The Spanish subjunctive", en Papers from the Eleventh Regional Meeting, Chicago Linguistic Society. Eds. R. E. Grossman, L. James San \& T. J. Vance. The Chicago Linguistic Society, Chicago, pp. 353-365.

Kuein, Philip 1974. Observations on the semantic of mood in Spanish. University of Washington (tesis doctoral inédita).

LamíQuiz, Vidal, y Miguel Ángel Pineda (eds.) 1983. Sociolingüística andaluza. T. 2: Material de encuestas para el estudio del habla urbana culta en Sevilla. Universidad, Sevilla.

Lavandera, Beatriz 1983. "Shifting moods in Spanish discourse", en Discourse perspectives on syntax. Ed. F. Klein-Andreu. Academic Press, New York, pp. 209-236.

Lope Blanch, JuAn M. 1958. "Algunos usos de indicativo por subjuntivo en oraciones subordinadas", NRFH, 12, 383-385.

— 1986. El estudio del español hablado culto. Historia de un proyecto, UNAM, México.

— (ed.) 1971. El habla de la ciudad de México. Materiales para su estudio. UNAM, México.

— (ed.) 1976. El habla popular de la ciudad de México. UNAM, México.

Marrone, Nila G. (ed.) 1992. El habla de la ciudad de La Paz. Materiales para su estudio. Ediciones Signo, La Paz.

Matluck, Joseph (ed.) s.a. Manuscrito inédito con 30 muestras de habla culta de La Habana.

Morales, Amparo, y María Vaquero (eds.) 1990. El habla culta de San Juan. Materiales para su estudio. Universidad de Puerto Rico, Río Piedras.

Otálora Fernández, Hilda, y Alonso González (eds.) 1986. El habla de la ciudad de Bogotá. Materiales para su estudio. Instituto Caro y Cuervo, Bogotá. 
Oviedo, Tito 1974. Mood and negation in Spanish noun clauses. University of California, Los Angeles (tesis doctoral inédita).

Porto Dapena, José Álvaro 1991. Del indicativo al subjuntivo. Arco/Libros, Madrid.

Quilis, Antonio, Manuel Esgueva, y Margarita Cantarero (eds.) 1981. El habla de la ciudad de Madrid. Materiales para su estudio. C.S.I.C., Madrid.

Rabanales, Ambrosio, y Lidia Contreras (eds.) 1974. El habla culta de Santiago de Chile. Materiales para su estudio. T. 1: Universidad de Chile, Santiago. T. 2: Instituto Caro y Cuervo, Bogotá, 1990.

Rosenblat, Ángel, y Paola Bentivoglio (eds.) 1979. El habla culta de Caracas. Materiales para su estudio. Universidad Central de Venezuela, Caracas.

Solano, Yamilet ca. 1994. El habla culta de San José, Costa Rica. Materiales para su estudio. Manuscrito inédito.

TARr, Frederick 1922. "Propositional complementary clauses in Spanish, with special reference to the works of Pérez Galdós", $R H i, 56,1-264$.

Terrell, Tracy D., \& JoAn Hooper 1974. "A semantically based analysis of mood in Spanish", $H, 57,484-494$.

Vogel, Lynn Cheryl 1979. A contribution to the study of the subjunctive in Spanish. University of California, Los Angeles (tesis doctoral inédita). 